\title{
GPx7 ameliorates non-alcoholic steatohepatitis by regulating oxidative stress
}

\author{
Hyeon Ju Kim ${ }^{1,2}$, Yoseob Lee, ${ }^{1,2}$, Sungsoon Fang ${ }^{2,3}$, Won Kim ${ }^{4}$, Hyo Jung Kim ${ }^{1, *}$ \& Jae-woo Kim ${ }^{1,2, *}$ \\ ${ }^{1}$ Department of Biochemistry and Molecular Biology, Chronic Intractable Disease for Systems Medicine Research Center, Yonsei University \\ College of Medicine, Seoul 03722, ${ }^{2}$ Brain Korea 21 PLUS Project for Medical Science, Yonsei University, Seoul 03722, ${ }^{3}$ Severance \\ Biomedical Science Institute, Yonsei University College of Medicine, Seoul 03722, ${ }^{4}$ Division of Gastroenterology and Hepatology, \\ Department of Internal Medicine, Seoul National University College of Medicine, Seoul Metropolitan Government Boramae Medical \\ Center, Seoul 07061, Korea
}

\begin{abstract}
Non-alcoholic fatty liver disease (NAFLD) is one of the most common liver diseases. NAFLD can further progress to irreversible liver failure such as non-alcoholic steatohepatitis (NASH) fibrosis and cirrhosis. However, specific regulator of NASHfibrosis has yet to be established. Here, we found that glutathione peroxidase 7 (GPx7) was markedly expressed in NASH fibrosis. Although GPx7 is an antioxidant enzyme protecting other organs, whether GPx7 plays a role in NASH fibrosis has yet to be studied. We found that knockdown of $G P x 7$ in transforming growth factor- $\beta$ (TGF- $\beta$ ) and free fatty acids (FFA)treated LX-2 cells elevated the expression of pro-fibrotic and pro-inflammatory genes and collagen synthesis. Consistently, $G P x 7$ overexpression in LX-2 cells led to the suppression of ROS production and reduced the expression of pro-fibrotic and pro-inflammatory genes. Further, NASH fibrosis induced by choline-deficient amino acid defined, high fat diet (CDAHFD) feeding was significantly accelerated by knockdown of $G P x 7$, as evidenced by up-regulated liver fibrosis and inflammation compared with CDAHFD control mice. Collectively, these results suggest that GPx7 might be a novel therapeutic target to prevent the progression and development of NAFLD. [BMB Reports 2020; 53(6): 317-322]
\end{abstract}

\section{INTRODUCTION}

Non-alcoholic fatty liver disease (NAFLD) is one of the most common liver dysfunctions (1). NAFLD encompasses a spectrum of liver diseases, ranging from simple steatosis to steatosis

${ }^{*}$ Corresponding authors. Jae-woo Kim, Tel: +82-2-2228-0837; Fax: +82-2-312-5041; E-mail: japol13@yuhs.ac; Hyo Jung Kim, Tel: +82-2-2228-0627; Fax: +82-2-312-5041; E-mail: hjkim17@yuhs.ac

https://doi.org/10.5483/BMBRep.2020.53.6.280

Received 11 November 2019, Revised 29 November 2019, Accepted 2 December 2019

Keywords: Gene therapy, GPx7, NAFLD, NASH, Oxidative stress combined with varying degrees of inflammation and fibrosis (2). The early stage of NAFLD involves simple steatosis, and is defined by a liver triglyceride content higher than $95 \%$ or by the triglyceride ratio in the cytoplasm $5 \%$ that of the liver $(3$, 4). It is reversible via lifestyle intervention, such as exercise (5) or caloric restriction (6). Hepatic steatosis can progress to non-alcoholic steatohepatitis (NASH) fibrosis, which can lead to end-stage liver disease $(2,3)$. NASH fibrosis is accompanied by ballooning degeneration, cell death, inflammation and deposition of fibrous tissue $(7,8)$. More than $20 \%$ of all patients with NASH fibrosis progress to cirrhosis, an irreversible disease $(9,10)$. Thus, the survival rate of chronic liver disease depends substantially on the successful treatment of $\mathrm{NASH}$ fibrosis. However, no effective pharmacological treatments are currently available for NASH fibrosis.

Simple steatosis triggered by continuous fat accumulation in the liver induces oxidation and production of pro-inflammatory cytokines and adipokines (adiponectin, leptin, tumor necrosis factor alphas (TNF $\alpha$ ), and interleukin 6 (IL-6)) (3). However, the mechanism of NASH fibrosis progression is largely unknown. Hepatic stellate cells (HSCs) are one of the common triggers of NASH fibrosis development. Activation of HSCs by various stimuli (e.g., transforming growth factor beta (TGF- $\beta))(11)$ that are transdifferentiated into myofibroblast-like cells strongly induces the secretion of fibrogenic mediators leading to excessive production of ECM proteins including collagen and alpha-smooth muscle actin ( $\alpha \mathrm{SMA})$ and release of inflammatory cytokines (12). Currently, many studies have shown that reactive oxygen species (ROS) might play a vital role in HSCs activation and proliferation (13). ROS production leads to NASH fibrosis via activation of HSCs $(14,15)$.

GPx7 is a member of the glutathione peroxidase family (GPx1-8), which is a major family of antioxidant enzymes that effectively control the cellular contents of hydrogen peroxidase $\left(\mathrm{H}_{2} \mathrm{O}_{2}\right)$ and organic hydroperoxide by reduced glutathione. Its main biological role is to protect the organism from oxidative damage (16). A recent study suggested that GPx7 is an oxidative stress sensor that transfers the ROS signals to its interacting proteins by shuttling disulfide bonds in response to

ISSN: 1976-670X (electronic edition)

Copyright (c) 2020 by the The Korean Society for Biochemistry and Molecular Biology

(ㄷ) This is an open-access article distributed under the terms of the Creative Commons Attribution Non-Commercial License (http://creativecommons.org/licenses/by-nc/4.0) which permits unrestricted non-commercial use, distribution, and reproduction in any medium, provided the original work is properly cited. 
various stress (17). Peng et al. have reported that GPx7 protects esophageal cells from bile acid-induced ROS production $(18,19)$ and oxidative DNA damage modulates the critical upstream regulator of inflammatory NF- $\mathrm{KB}$ signaling (20). However, the effects of GPx7 on NASH fibrosis remain unexplored.

Thus, in the present study, we investigated whether GPx7 expression is associated with the development of NASH fibrosis using in vitro and in vivo models. Here, we found that GPx7 was significantly upregulated in NASH fibrosis compared with normal and simple models of steatosis. Moreover, GPX7 overexpression ameliorated NASH fibrosis via reduction of cellular ROS synthesis and regulation of pro-fibrotic and proinflammatory genes. In addition, GPx7 has been implicated in CDAHFD feeding-induced NASH fibrosis in vivo. Taken together, our findings indicate that GPx7 represents a promising target for the development of effective prophylactic and therapeutic strategy against NASH fibrosis.

\section{RESULTS}

GPx7 is highly expressed in non-alcoholic steatohepatitis To identify the specific regulator of NASH fibrosis, we conducted RNA sequencing using in vitro and in vivo models of simple steatosis and NASH fibrosis. Simple models of steatosis were the oleic acid (OA)-treated Hepa1-6 cells and high fat diet (HFD)-fed mice. NASH fibrosis was induced by TGF- $\beta$ and FFA in LX-2 cells and CDAHFD in mice. In RNA-seq, we obtained several genes, which were expressed more than two-fold higher in NASH fibrosis than in simple steatosis (Fig. 1A). Among these genes, only GPx7 expression was highly increased in NASH fibrosis-specific manner. The expression level of each GPx isoform in simple steatosis and NASH fibrosis was confirmed using RT-PCR (Fig. 1B). Consistent with RNA-seq results, GPx7 expression was sig-
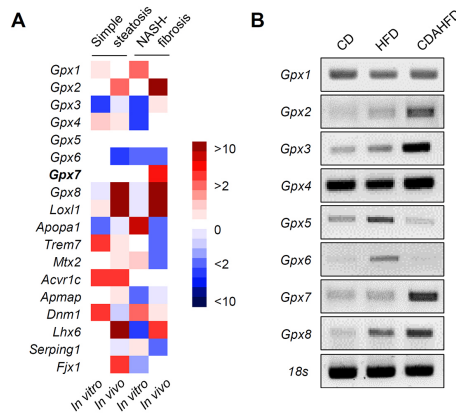

C

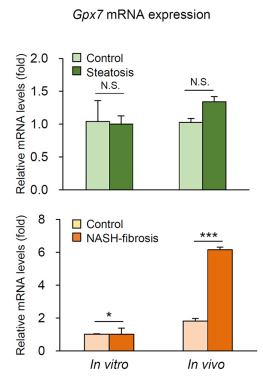

Fig. 1. GPX7 is highly expressed in NASH fibrosis. (A) Selected genes from RNA-seq. (B) Relative mRNA expression of GPx family in indicated mice liver. CD, chow diet; HFD, high-fat diet; CDAHFD, choline-deficient, amino-acid, high-fat diet. (C) Real-time PCR of GPX7 expression in simple steatosis and NASH fibrosis. All data are presented \pm S.D. ${ }^{* P}<0.05, * * P<0.01$, ***P $<0.001$ nificantly increased only in NASH fibrosis, but not in simple steatosis (Fig. 1C). These results suggest that increased GPx7 expression in NASH fibrosis may mediate the pathological mechanism of NASH fibrosis.

\section{GPx7 plays a critical role in NASH fibrosis progression regulating oxidative stress}

Next, to evaluate whether GPx7 affects NASH fibrosis, GPx7 was knocked down in LX-2 cells using siRNA. LX-2 cells represent hepatic stellate cell models, which are commonly used for the NASH fibrosis model in vitro. As shown in Fig. 2A and $B$, fibrosis- and inflammation-related genes were significantly upregulated in GPx7-siRNA-transfected LX-2 cells. In Sirius Red staining, the knockdown of GPx7 induced collagen deposition (Fig. 2C). These results suggest that inhibition of GPx7 aggravates the progression of NASH fibrosis. Consistently, GPx7 overexpression in LX-2 cells decreased pro-fibrotic and pro-inflammatory gene expression (Fig. 2D). Since GPx7 has been reported as an antioxidant against oxidative stress (21), we measured the cellular ROS production in GPx7overexpressed LX-2 cells to confirm the status of oxidative stress. As expected, the overexpression of GPx7 in LX-2 cells reduced fluorescence intensity of DCF-DA, indicating decreased ROS production (Fig. 2E). These results indicate that GPx7 inhibits NASH fibrosis progression by suppressing cellular levels of oxidative stress.

\section{Knockdown of GPx7 accelerates the progression of liver fibrosis in CDAHFD-fed mice}

To investigate whether the GPx7 deficiency promotes NASH

A

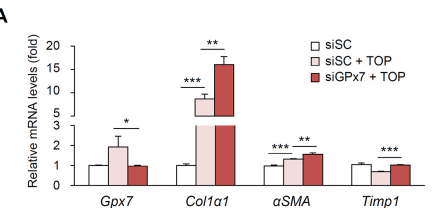

B c

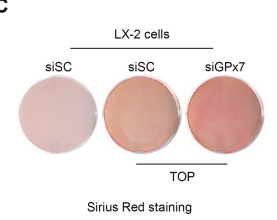

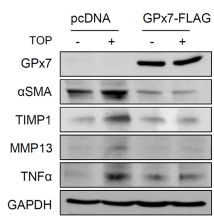
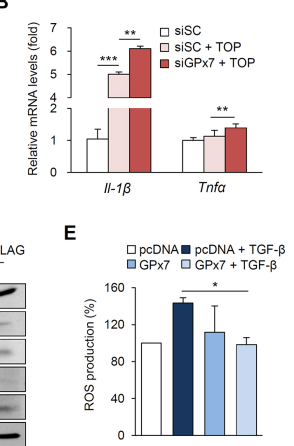

Fig. 2. GPx7 prevents NASH fibrosis by suppressing ROS production. (A, B) Relative mRNA expression of indicated genes in LX-2 cells. Cells transfected with either negative control siRNA or si-GPx7 were treated with TOP. TOP, TGF- $\beta$ (3 ng) and FFA (1 mM OA, PA). (C) Sirus Red staining of LX-2 cells. Collagen, red; muscle fiber, yellow. (D) Cells transfected with the pcDNA3.0 or pcDNA3-GPx7-FLAG vector. Western blotting analysis using indicated antibodies. GAPDH was used as the loading control. (E) ROS production using flow cytometry of GPx7 overexpressed LX-2 cells. All data are presented \pm S.D. ${ }^{*} \mathrm{P}<0.05, * * \mathrm{P}<0.01$, $* * * P<0.001$ 
fibrosis in vivo, we developed hepatic GPx7-deficient mice using an adenovirus containing short-hairpin RNA of GPx7 (Ad-shGPx7). After three weeks of feeding with CDAHFD, the C57BL/6 mice were injected Ad-shGPx7 and control virus via tail-vein, and the mice were sacrificed seven days later (Fig. 3A). Ad-shGPx7 had no effect on body weight in the CDAHFD group (Fig. 3B). However, hematoxylin \& eosin (H\&E) staining and Masson's trichrome (MTC) staining showed that CDAHFD increased collagen deposition and NASH fibrosis was aggravated by GPx7 knockdown in liver (Fig. 3C). Furthermore, disproportionate to the body weight, the liver size was increased in Ad-shGPx7-injected mice (Fig. 3D), as evidenced by the increased ratio of liver weight and liver to body weight (Fig. 3E). In addition, the knockdown of GPx7 led to increased serum aspartate transaminase (AST) and alanine transaminase (ALT) levels, which are indicators of liver inflammation (Fig. $3 F)$, suggesting that GPx7 is required for protection against hepatic fibrosis and inflammation. However, GPx7 knockdown did not affect TG accumulation in serum or liver (Fig 3G).

To verify the GPx7 reduction and NASH fibrosis-related gene expression, RT-PCR was performed using liver samples. The GPx7 expression was significantly induced by CDAHFD feeding and reduced after Ad-shGPx7 injection (Fig. 4A). Consistent with GPX7 reduction, pro-fibrotic and pro-inflammatory gene expression was dramatically increased in CDAHFD-fed mice liver (Fig. 4B and C). These changes were confirmed at the protein level (Fig. 4D). Thus, the knockdown of GPx7 accelerated CDAHFD-induced NASH fibrosis.
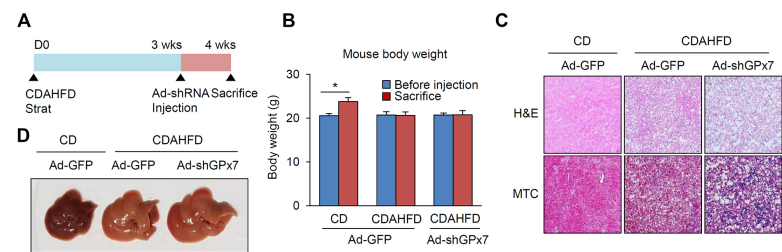

E $\mathbf{F}$
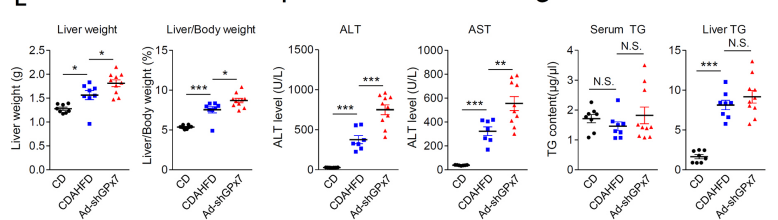

Fig. 3. Knockdown of GPx7 induces NASH fibrosis. (A) Schematic diagram of in vivo experiments. (Chow diet, $n=8$; CDAHFD group and sh-GPX7 group, $\mathrm{n}=10$ ). (B) Body weight changes in mice. (C) H\&E and MTC staining of liver sections derived from mice. Collagen, blue; muscle fiber, red. (D) Representative liver tissue image. (E) Liver weight and the ratio of liver to body weight in mice. (F) Serum ALT and AST level in mice. (G) Serum TG and liver TG level in mice. Black-filled circle, CD + Ad-shControl; Blue-filled square, CDAHFD + Ad-shControl; Red-filled triangle, CDAHFD + Ad-shGPx7. All data are presented \pm S.D. ${ }^{* P}<0.05$, $* * P<0.01, * * * P<0.001$.

\section{DISCUSSION}

NASH fibrosis is the result of chronic liver injury induced by persistent fat accumulation of several cytokines via the interaction between HSCs and Kupffer cells, and promotion of ECM synthesis by activated HSCs (22). NASH fibrosis is characterized by excessive collagen accumulation, lobular and portal inflammation, hepatic ballooning and fibrosis (23). Moreover, NASH fibrosis acts as an indicator of progressive molecular pathogenesis and inflammatory reaction (24). Liver cell damage triggered by various causes induces hepatocellular carcinoma (HCC) and hepatocyte dysfunction (25). Thus, the survival rates of chronic liver disease strongly rely on the diagnosis and treatment of NASH fibrosis, which leads to irreversible cirrhosis. Nevertheless, a therapeutic strategy against $\mathrm{NASH}$ fibrosis has yet to be established. Therefore, it is important to identify new regulators to develop effective strategies for preventing NASH fibrosis progression. Here, we found that GPx7 may play an important role during NASH fibrosis via regulation of liver fibrosis-related gene expression and oxidative stress.

HSC activation via induction of TGF- $\beta$ causes NASH fibrosis by accumulating ECM (26-28). High-fat diet (HFD) is usually used in mouse models of liver injury-mediated HSC activation $(29,30)$. In this study, CDAHFD used to induce NASH fibrosis is composed of $60 \mathrm{kcal} \%$ fat and $0.1 \%$ methionine, without containing choline (30). Although fat accumulation accompanied by inflammation and fibrosis is a key characteristic of NASH, our results showed no changes in serum TG levels due to CDAHFD inhibition of VLDL secretion from liver to serum. However, CDAHFD induced liver TG accumulation in mice and the accumulated TG level was not altered by knockdown

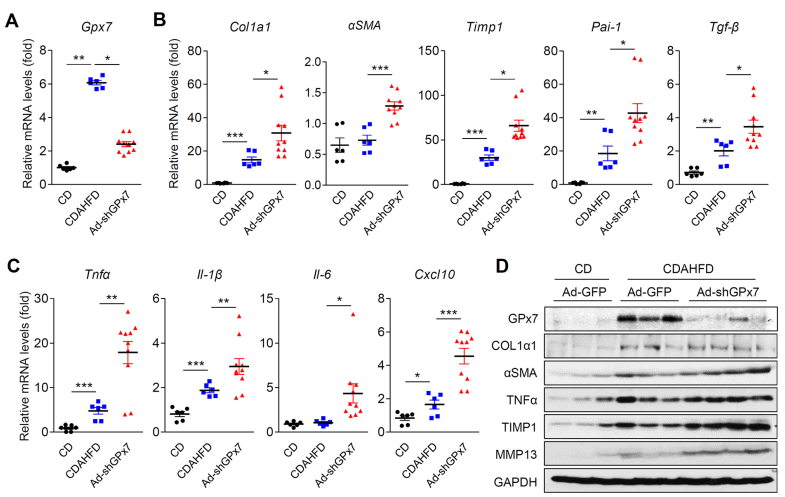

Fig. 4. Knockdown of GPx7 increases hepatic inflammation and fibrosis-related gene expression. (A) Relative mRNA expression of GPx7. (B, C) Relative mRNA levels of indicated genes. (D) Western blotting analysis using indicated antibodies. GAPDH was used as the loading control. Black-filled circle, CD + Ad-shControl; Bluefilled square, CDAHFD + Ad-shControl; Red-filled triangle, CDAHFD + Ad-shGPx7. All data are presented \pm S.D. ${ }^{*} \mathrm{P}<0.05, * * \mathrm{P}<$ $0.01, * * * \mathrm{P}<0.001$. 
of GPx7, suggesting that GPx7 was not involved in fat accumulation during NASH progression. Thus, GPx7 may regulate $\mathrm{NASH}$ progression via fibrosis rather than simple steatosis.

Oxidative stress has been reported to play a predominant pro-fibrotic role in liver fibrosis $(13,31)$. GPx7 is a known regulator of ROS-dependent oxidative DNA damage in several cancer types, such as gastric and esophageal cancers (17). However, the exact role of GPx7 in liver disease is not shown. In this study, the antioxidant action of GPx7 in liver was confirmed by the NASH fibrosis model. To our knowledge, this is the first report demonstrating the function of GPX7 in $\mathrm{NASH}$ fibrosis. HSC activation triggered GPx7 overexpression and GPx7 modulated pro-fibrotic gene expression via regulation of intracellular ROS levels. This result indicated that oxidative stress contributes to liver fibrosis during NASH progression, suggesting that antioxidants may represent therapeutic alternatives in NASH fibrosis.

Overall, our findings demonstrate that GPx7 effectively ameliorates liver fibrogenesis via inhibition of the oxidative stress. GPX7 exhibited a potent anti-fibrotic effect by reducing inflammatory cytokine production, improving pathological changes, and inhibiting ROS production. Taken together, GPx7 overexpression might represent a potential therapeutic target in NASH fibrosis.

\section{MATERIALS AND METHODS}

\section{Experimental animals}

Male C57BLJ mice were purchased from Japan SLC, Inc (Shinzuoka, Japan). The animals were maintained in a temperature-controlled room $\left(22^{\circ} \mathrm{C}\right)$ on a $12: 12$-h light-dark cycle. Six-week-old mice were fed a normal chow-diet for normal control model, and CDAHFD (Choline-deficient, L-amino aciddefined, high-fat diet, Research Diets, New Brunswick, NJ, USA) for 3 weeks to develop the NASH fibrosis model. Nineweek-old male C57BL/6 mice were injected with adenoviruscontaining short-hairpin RNA (shRNA) or control recombinant adenovirus. Recombinant adenoviruses $\left(1 \times 10^{9}\right.$ pfu) were delivered by tail-vein-injection. After seven days, mice are sacrificed, securing the liver. All procedures were approved by the Committee on Animal Investigations of Yonsei University.

\section{Cell culture}

LX-2 cells (human stellate cell line) were maintained in Dulbecco's Modified Eagle Medium (DMEM; Invitrogen, Carlsbad, CA, USA) containing $100 \mathrm{U} / \mathrm{mL}$ penicillin, 100 $\mu \mathrm{l} / \mathrm{mL}$ streptomycin, supplemented with heat-inactivated $10 \%$ fetal bovine serum (FBS; Invitrogen) at $37^{\circ} \mathrm{C}$, in an atmosphere of $95 \%$ air and $5 \% \mathrm{CO}_{2}$.

\section{Sirius Red Staining}

Sirius Red Staining was used to observe fibrosis in cells. Cells were washed in distilled water, and fixed in $70 \%$ cold ethanol for $15 \mathrm{~min}$. The staining solution was applied to completely cover the tissue section and incubated for $60 \mathrm{~min}$. The staining solution was removed and cells were rinsed twice with acetic acid solution and absolute alcohol.

\section{FACS analysis}

LX-2 cells were transfected with control or GPx7-overexpressing vector. After $24 \mathrm{~h}$, cells were treated with ROS stimulator and $5 \mathrm{ng} / \mu \mathrm{l} \mathrm{TGF}-\beta$, and after $30 \mathrm{~min}$ supplemented with 10 $\mu \mathrm{M}$ H2DCFDA for $15 \mathrm{~min}$. The cells were trypsinized and collected by centrifugation, washed with $1 \times$ PBS twice. Labeled cells were analyzed using a FACS caliber flow cytometry system, and data were analyzed using the ModFit software (BD Biosciences).

\section{Preparation of recombinant adenovirus}

Murine GPx7 was obtained by reverse transcriptase-polymerase chain reaction (RT-PCR) with total RNA obtained from C57BL/6 mice. Recombinant adenovirus, containing the murine GPx7 gene under the control of the CMV promoter, was prepared. An E1 shuttle vector expressing murine GPx7 was first constructed and then linearized with Pmel digestion. The linearized shuttle vector was then cotransformed into Escherichia coli BJ5183 along with pAdEasy1 vector. The virus was propagated in 293A cells and purified by $\mathrm{CsCl}$ density purification, dissolved in $1 \times \mathrm{PBS}$ and stored at $-80^{\circ} \mathrm{C}$. Viral particle numbers were calculated from the measurements of absorbance at $260 \mathrm{~nm}\left(\mathrm{~A}_{260}\right)$. The multiplicity of infection (MOI) was calculated from viral particle number. A recombinant adenovirus containing the GFP gene was used as a control.

\section{Measurement of triglyceride level in the hepatocytes}

Lipid extracts were prepared by homogenizing $0.2 \mathrm{~g}$ of liver in chloroform/methanol $(2: 1, \mathrm{v} / \mathrm{v})$ with a final volume of $4 \mathrm{~mL}$. The homogenate was incubated with vortexing for $10 \mathrm{~min}$, followed by the addition of $0.8 \mathrm{~mL}$ of $50 \mathrm{mM} \mathrm{NaCl}$. The mixture was vortexed for $10 \mathrm{~min}$, and then centrifuged at $4^{\circ} \mathrm{C}$ for $10 \mathrm{~min}$. The organic phase $(25 \mu \mathrm{l}$ of lower layer) was transferred and $25 \mu \mathrm{l}$ of Triton X-100/chloroform (7.5:17.5, $\mathrm{v} / \mathrm{v}$ ) was added to samples and vortexed for $10 \mathrm{~min}$. The solvents were vaporized with a vacuum evaporator. TG concentrations were determined using $25 \mu \mathrm{l}$ of extract in a commercial colorimetric assay (Thermo scientific, Waltham, MA, USA). Samples and standards were vortexed and incubated at $37^{\circ} \mathrm{C}$ for $30 \mathrm{~min}$. The TG level were calculated from measurements of absorbance at $500 \mathrm{~nm}$ and expressed as mg TG/g liver wet weight.

\section{Histological analysis}

The liver tissues were fixed in $10 \%$ neutral-buffered formalin. Following fixation, the liver was trimmed, cryoembedded, sectioned, and stained with hematoxylin and eosin (H\&E) (32) and masson's trichrome. 


\section{Gene expression analysis}

Total RNA was isolated using TRlzol reagent (Invitrogen, Carlsbad, CA, USA) according to the manufacturer's instructions. For RT-PCR, cDNA was synthesized from $5 \mu$ g of total RNA using random hexamer primers and Superscript reverse transcriptase III (Invitrogen). Real-time PCR was conducted using SYBR Green Master mix (Applied Biosystems, Foster city, CA, USA) in a total volume of $20 \mu \mathrm{l}$. Transcripts were detected by real-time qPCR with a Step One instrument (Applied Biosystems). All data were normalized to $18 \mathrm{~S}$ and quantitative measures, and were obtained using the $\Delta \Delta-\mathrm{Ct}$ method. All reactions were perfomed in duplicate. The relative expression levels and S.D. values were calculated using the comparative method.

\section{Transient transfection assay}

Cells were plated on 60-mm diameter dishes $24 \mathrm{~h}$ before transfection. The following double-stranded stealth siRNA oligonucleotides (Snata Cruz Biotechnologh) were used: mouse GPx7 siRNA oligonucleotides (sc-78832). Control oligonucleotides with comparable GC content were also obtained from Invitrogen. For knockdown, cells were transfected with control or gene-specific siRNA at $60 \mathrm{nM}$ in OPTI-MEM medium using Lipofectamine RNAiMAX (Invitrogen), according to the manufacturer's protocol. The next day, the medium was replaced with fresh DMEM containing 10\% FBS, $100 \mathrm{U} / \mathrm{mL}$ penicillin and $100 \mu \mathrm{g} / \mathrm{mL}$ streptomycin, and the cells were incubated for $24 \mathrm{~h}$ before harvest. Total RNA extracts were prepared from the cell at the indicated time point ( 2 days), and RT-PCR or real-time PCR was performed. For the overexpression, the cells were plated at a density of $5 \times 10^{5}$ cells/well. The plasmid, $\mathrm{GPx} 7$ overexpressing vector (pcDNA3-GPx7-FLAG) was packaged with Lipofectamine 2000 (Invitrogen), and cells were transfected with either a control or GPx7-overexpressing vector according to the manufacturer's instructions.

\section{Western blot analysis and antibodies}

For protein analysis, cells were washed with $1 \times$ PBS and lysed in a buffer containing $1 \%$ sodium dodecyl sulfate (SDS) and 60 $\mathrm{mM}$ Tris- $\mathrm{Cl}$, $\mathrm{pH}$ 6.8, and tissues were lysed in PRO-PREP ${ }^{\mathrm{TM}}$ (iNtRON Biotechnology). The lysate was mixed briefly using a vortex, boiled for $10 \mathrm{~min}$, and centrifuged at 13,000 $\mathrm{g}$ for 10 $\min$ at $4^{\circ} \mathrm{C}$. Protein concentrations were assessed using the BCA assay kit (Pierce). Protein samples of equal amount were separated by SDS-PAGE and transferred to nitrocellulose membranes. Immunoblot analysis were performed using the following antibodies: polyclonal antibodies against GPX7 (Proteintech), Col1 $\alpha 1$ (Abcam), TNF $\alpha$ (Cell Signaling), $\alpha$ SMA, TIMP1, MMP13, ADRP, and GAPDH (Santa Cruz Biotechnology).

\section{Statistical analysis}

All results are expressed as mean \pm S.D. Statistical comparisons of groups were made using an unpaired Student's test.

\section{ACKNOWLEDGEMENTS}

This work was supported by the National Research Foundation of Korea (NRF) grant funded by the Korean Government, Ministry of Science and ICT (MSIT) (NRF-2018R1A5A2025079).

\section{CONFLICTS OF INTEREST}

The authors have no conflicting interests.

\section{REFERENCES}

1. Buzzetti E, Pinzani M and Tsochatzis EA (2016) The multiple-hit pathogenesis of non-alcoholic fatty liver disease (NAFLD). Metabolism 65, 1038-1048

2. Hardy T, Oakley F, Anstee QM and Day CP (2016) Nonalcoholic Fatty Liver Disease: Pathogenesis and Disease Spectrum. Annu Rev Pathol 11, 451-496

3. Cohen JC, Horton JD and Hobbs HH (2011) Human fatty liver disease: old questions and new insights. Science $332,1519-1523$

4. Szczepaniak LS, Nurenberg P, Leonard D et al (2005) Magnetic resonance spectroscopy to measure hepatic triglyceride content: prevalence of hepatic steatosis in the general population. Am J Physiol Endocrinol Metab 288, E462-468

5. Promrat K, Kleiner DE, Niemeier HM et al (2010) Randomized controlled trial testing the effects of weight loss on nonalcoholic steatohepatitis. Hepatology 51, 121-129

6. Huang MA, Greenson JK, Chao C et al (2005) One-year intense nutritional counseling results in histological improvement in patients with non-alcoholic steatohepatitis: a pilot study. Am J Gastroenterol 100, 1072-1081

7. Tacke $F$ and Trautwein C (2015) Mechanisms of liver fibrosis resolution. J Hepatol 63, 1038-1039

8. Bataller R and Brenner DA (2005) Liver fibrosis. J Clin Invest 115, 209-218

9. Jeong SH and Lim DS (2018) Insulin receptor substrate 2: a bridge between Hippo and AKT pathways. BMB Rep 51, 209-210

10. Stal $P$ (2015) Liver fibrosis in non-alcoholic fatty liver disease - diagnostic challenge with prognostic significance. World J Gastroenterol 21, 11077-11087

11. Yang KL, Chang WT, Hong MY, Hung KC and Chuang CC (2017) Prevention of TGF-beta-induced early liver fibrosis by a maleic acid derivative anti-oxidant through suppression of ROS, inflammation and hepatic stellate cells activation. PLoS One 12, e0174008

12. Iwaisako K, Brenner DA and Kisseleva T (2012) What's new in liver fibrosis? The origin of myofibroblasts in liver fibrosis. J Gastroenterol Hepatol 27 Suppl 2, 65-68

13. Gandhi CR (2012) Oxidative Stress and Hepatic Stellate Cells: A PARADOXICAL RELATIONSHIP. Trends Cell Mol Biol 7, 1-10

14. Torok NJ (2016) Dysregulation of redox pathways in liver fibrosis. Am J Physiol Gastrointest Liver Physiol 311, G667G674

15. Sanchez-Valle V, Chavez-Tapia NC, Uribe M and MendezSanchez N (2012) Role of oxidative stress and molecular 
changes in liver fibrosis: a review. Curr Med Chem 19, 4850-4860

16. Muthukumar K and Nachiappan V (2010) Cadmiuminduced oxidative stress in Saccharomyces cerevisiae. Indian J Biochem Biophys 47, 383-387

17. Chen YI, Wei PC, Hsu JL, Su FY and Lee WH (2016) NPGPx (GPx7): a novel oxidative stress sensor/transmitter with multiple roles in redox homeostasis. Am J Transl Res 8, 1626-1640

18. Peng D, Belkhiri A, Hu T et al (2012) Glutathione peroxidase 7 protects against oxidative DNA damage in oesophageal cells. Gut 61, 1250-1260

19. Peng D, Hu T, Soutto M, Belkhiri A, Zaika A and El-Rifai W (2014) Glutathione peroxidase 7 has potential tumour suppressor functions that are silenced by location-specific methylation in oesophageal adenocarcinoma. Gut 63, 540-551

20. Peng DF, Hu TL, Soutto M, Belkhiri A and El-Rifai W (2014) Loss of glutathione peroxidase 7 promotes TNF-alpha-induced NF-kappaB activation in Barrett's carcinogenesis. Carcinogenesis 35, 1620-1628

21. Mehmeti I, Lortz S, Avezov E, Jorns A and Lenzen S (2017) ER-resident antioxidative GPx7 and GPx8 enzyme isoforms protect insulin-secreting INS-1E beta-cells against lipotoxicity by improving the ER antioxidative capacity. Free Radic Biol Med 112, 121-130

22. Fujita T and Narumiya S (2016) Roles of hepatic stellate cells in liver inflammation: a new perspective. Inflamm Regen 36, 1
23. Benedict $M$ and Zhang X (2017) Non-alcoholic fatty liver disease: An expanded review. World J Hepatol 9, 715-732

24. Del Campo JA, Gallego P and Grande L (2018) Role of inflammatory response in liver diseases: Therapeutic strategies. World J Hepatol 10, 1-7

25. Lo RC and Kim H (2017) Histopathological evaluation of liver fibrosis and cirrhosis regression. Clin Mol Hepatol 23, 302-307

26. Higashi T, Friedman SL and Hoshida Y (2017) Hepatic stellate cells as key target in liver fibrosis. Adv Drug Deliv Rev 121, 27-42

27. Safadi R and Friedman S (2002) Hepatic fibrosis-role of hepatic stellate cell activation. MedGenMed 4, 27-27

28. Friedman SL, Neuschwander-Tetri BA, Rinella $M$ and Sanyal AJ (2018) Mechanisms of NAFLD development and therapeutic strategies. Nat Med 24, 908-922

29. Zhou Z, Xu MJ, Cai Y et al (2018) Neutrophil-Hepatic Stellate Cell Interactions Promote Fibrosis in Experimental Steatohepatitis. Cell Mol Gastroenterol Hepatol 5, 399-413

30. Jing XY, Yang XF, Qing K and Ou-yang Y (2013) Roles of the lipid metabolism in hepatic stellate cells activation big up tri, open. Chin Med Sci J 28, 233-236

31. Novo E, Cannito S, Paternostro C, Bocca C, Miglietta A and Parola M (2014) Cellular and molecular mechanisms in liver fibrogenesis. Arch Biochem Biophys 548, 20-37

32. Lee DH, Lee B, Park JS et al (2019) Inactivation of Sirtuin2 protects mice from acetaminophen-induced liver injury: possible involvement of ER stress and S6K1 activation. BMB Rep 52, 190-195 